\title{
Topologically ordered phases of smectics confined in anisotropic random media: smectic Bragg glasses
}

\author{
Karl Saunders $\dagger$, Brad Jacobsen $\ddagger$, Leo Radzihovsky $\ddagger$ and John Toner $\dagger$ \\ $\dagger$ Department of Physics, Materials Science Institute, and Institute of Theoretical Science, \\ University of Oregon, Eugene, OR 97403, USA \\ $\ddagger$ Department of Physics, University of Colorado, Boulder, CO 80309, USA
}

\begin{abstract}
We report the existence of two new topologically ordered glass phases of smectics in strained aerogel. In contrast to the case of unstrained aerogel, we find compelling theoretical arguments that a smectic in uniaxially stretched aerogel exhibits, for homeotropic nematic-aerogel alignment, a 'smectic Bragg glass' in the universality class of the ' $X Y$ Bragg glass'. On the other hand, a uniaxial compression, with homeotropic alignment, leads to an entirely novel type of anisotropic smectic elastic glass phase that we call the ' $m=1$ Bragg glass'. This latter phase exhibits anomalous elasticity, characterized by exponents that we calculate to high precision. We present a phase diagram for the system in the aerogel density-strain parameter space, which should be accessible experimentally. We also make numerous other scaling predictions for experimentally observable quantities.
\end{abstract}

The effect of confining liquid crystals to random porous structures continues to be a fascinating area of active research [1]. It was shown in a recent theoretical analysis that arbitrarily weak quenched disorder arising from such confinements destroys conventional (quasi-) long-ranged translational smectic order in 3D [2]. A translationally disordered but topologically ordered 'smectic Bragg glass' (SBG) phase was proposed as the new thermodynamically distinct lowtemperature phase in these smectic systems. Unfortunately, it proved impossible to make a compelling theoretical argument for the stability of such a glass phase in quenched random isotropic structures, e.g. aerogel.

We show in this article that such a compelling argument can be made for smectics in a uniaxially strained aerogel, which certainly exhibit two types of low- $T$ BG phase, that are thermodynamically distinct from the high- $T$ nematic (or perhaps 'nematic elastic glass' (NEG)) and isotropic liquid phases. For parallel nematogen-surface alignment (assumed throughout), a stretch (figure 1(a)) of the aerogel will lead to an ' $X Y \mathrm{BG}$ ' in the isotropic universality class of randomly pinned vortex lattices, CDWs, and random-field $X Y$ magnets ( $\operatorname{RF} X Y$ ) [3], while a compression (figure 1(b)) will lead to a novel ' $m=1 \mathrm{BG}$ ', with triaxially anisotropic scaling, that should be similar to that of a discotic in isotropic aerogel [4]. For homeotropic alignment, the phases reverse with respect to stretch and compression.

We predict two possible low, constant- $T$ phase diagrams, depending on whether the SBG is stable (figure 2(b)) or not (figure 2(a)).

Recent experiments [5] suggest the former possibility. The loci of the phase boundaries in figure 2(a), for small strain, $\sigma$, are universal and satisfy

$$
\Delta(\sigma) \propto\left(K^{3} B\right)^{1 / 2}(\sigma / B)^{\rho}
$$




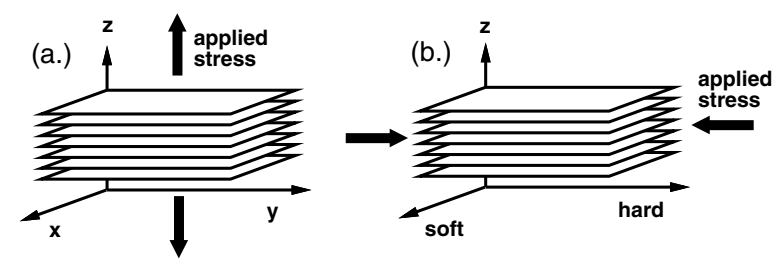

Figure 1. (a) Stretch along the $\hat{z}$-direction. (b) Compression along the $\perp$ direction.
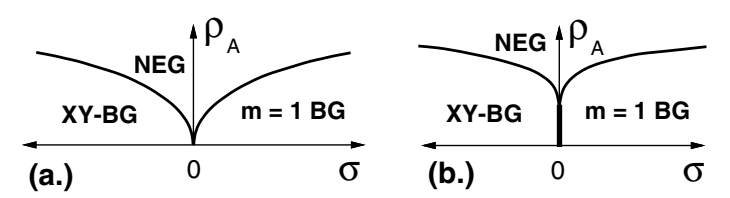

Figure 2. Two possible phase diagram topologies, depending on whether SBG is stable for isotropic confinement $(\sigma=0)$.

where $\sigma$ is proportional to the uniaxial stress applied to the aerogel fibres, $\Delta$ is a measure of the tilt disorder, $B$ and $K$ are bulk smectic elastic moduli, and $\rho$ is a universal exponent expressible in terms of anomalous elasticity exponents $\tilde{\eta}_{B}$ and $\tilde{\eta}_{K}$ for unstrained aerogel. Our best estimate is $\rho \approx 2 / 5$ in $3 \mathrm{D}$ [2].

Our model of the smectic in aerogel treats the local smectic layer displacement $u(r)$ and the local nematic director $\hat{\boldsymbol{n}}(\boldsymbol{r})$ as the only important fluctuating quantities, ignoring fluctuations in the magnitude $|\psi|$ of the smectic order parameter $\psi=|\psi| \mathrm{e}^{-\mathrm{i} q_{0} u(r)}$ about its mean $\left|\psi_{0}\right|$.

The important effects of the aerogel are completely described by only two disorder types. One is the random-field translational disorder

$$
\delta H_{r f}=\Re \int \mathrm{d}^{d} \boldsymbol{r}\left|\psi_{0}\right| V(\boldsymbol{r}) \mathrm{e}^{\mathrm{i} q_{0} u(\boldsymbol{r})}
$$

coupling to $u(\boldsymbol{r})$, with $V(\boldsymbol{r})$ a complex random potential which at long length scales can be accurately represented as zero-mean and short-ranged [2] Gaussian statistics with $\overline{V(\boldsymbol{r}) V^{*}\left(\boldsymbol{r}^{\prime}\right)}=\tilde{\Delta}_{V} \delta^{d}\left(\boldsymbol{r}-\boldsymbol{r}^{\prime}\right)$. The other type of disorder is the random-tilt orientational disorder given by

$$
\delta H_{t}=-\int \mathrm{d}^{d} \boldsymbol{r}(\boldsymbol{g}(\boldsymbol{r}) \cdot \hat{\boldsymbol{n}})^{2}
$$

describing the tendency of nematogens $\hat{\boldsymbol{n}}(\boldsymbol{r})$ to align along the local aerogel strand directed along $\boldsymbol{g}(\boldsymbol{r})$, and at long length scales is completely described by short-ranged [2] correlations $\overline{g_{i}(\boldsymbol{r}) g_{j}\left(\boldsymbol{r}^{\prime}\right)}=(1 / 2)\left(\sqrt{\Delta} \delta_{i j}-\gamma e_{i} e_{j}\right) \delta^{d}\left(\boldsymbol{r}-\boldsymbol{r}^{\prime}\right)$, with $\hat{\boldsymbol{e}}$ the uniaxial direction (i.e., the axis of the strain applied to the aerogel). In the above, $\tilde{\Delta}_{V}=\Gamma_{u}\left(a_{f} / L_{f}\right)^{d-d_{f}}\left(1 /\left(a_{f} q_{0}\right)\right)$, $\Delta=\Gamma_{n}\left(a_{f} / L_{f}\right)^{d-d_{f}}, d_{f}$ is the aerogel's fractal dimension for length scales $a_{f}<r<L_{f}$, and $\Gamma_{u}, \Gamma_{n}, \gamma$ are phenomenological parameters, with $\gamma$ the anisotropy parameter which at small strains is proportional to $\sqrt{\Delta}$ and the stress $\sigma$ applied to the aerogel. $\gamma<0$ for a stretch illustrated in figure 1(a).

Assuming (as we will verify a posteriori) that fluctuations in $\hat{\boldsymbol{n}}$ from a perfect alignment with the smectic layer normal (taken along $\hat{\boldsymbol{z}}$ ) are small allows us to integrate $\hat{\boldsymbol{n}}$ out of the partition function, with the only effect being the replacement $\delta \boldsymbol{n} \rightarrow \nabla_{\perp} u$ [2]. The resulting 
Hamiltonian is given by

$$
\begin{aligned}
H=\int \mathrm{d}^{d} \boldsymbol{r}[ & \frac{K}{2}\left(\nabla_{\perp}^{2} u\right)^{2}+\frac{B}{2}\left(\partial_{z} u-\frac{1}{2}\left(\nabla_{\perp} u\right)^{2}\right)^{2} \\
& +\left(g_{z}(\boldsymbol{r}) \boldsymbol{\nabla}_{\perp} u\right)^{2}-\left(\boldsymbol{g}(\boldsymbol{r}) \cdot \boldsymbol{\nabla}_{\perp} u\right)^{2}-2 g_{z}(\boldsymbol{r}) \boldsymbol{g}(\boldsymbol{r}) \cdot \boldsymbol{\nabla}_{\perp} u \\
& \left.-\left|\psi_{0}\right| \Re\left\{V(\boldsymbol{r}) \mathrm{e}^{\mathrm{i} q_{0} u(\boldsymbol{r})}\right\}\right] .
\end{aligned}
$$

The form of the anharmonic elastic terms is dictated by the underlying invariance of the bulk smectic phase under rotations about any axis lying in the $\boldsymbol{r}_{\perp}$-plane.

After introducing $n$ replica fields $u_{\alpha}$ and integrating out the disorder, we obtain the Hamiltonian whose form strongly depends on the type of uniaxial strain. Stretching the aerogel strands will cause the layer normal, $\hat{z}$, to align with $\hat{e}$ (figure 1(a)). Smectics confined inside this structure, to harmonic order in elasticity (with elastic anharmonicity irrelevant), are described by

$$
\begin{array}{rl}
H_{X Y}=\frac{1}{2} \int \mathrm{d}^{d} & \boldsymbol{r} \sum_{\alpha=1}^{n}\left[K\left(\nabla_{\perp}^{2} u_{\alpha}\right)^{2}+B\left(\partial_{z} u_{\alpha}\right)^{2}+|\gamma|\left(\nabla_{\perp} u_{\alpha}\right)^{2}\right] \\
& -\frac{1}{2 T} \int_{r} \sum_{\alpha, \beta=1}^{n}\left[(\Delta+\sqrt{\Delta}|\gamma|)\left(\nabla_{\perp} u_{\alpha}\right) \cdot\left(\nabla_{\perp} u_{\beta}\right)+\Delta_{V} \cos \left[q_{0}\left(u_{\alpha}-u_{\beta}\right)\right]\right]
\end{array}
$$

where $\Delta_{V}=2\left|\psi_{0}\right|^{2} \tilde{\Delta}_{V}$. At length scales smaller than a crossover length scale $\xi_{\perp}^{c}$ (to be defined below), the behaviour is that of a smectic pinned by isotropic unstrained aerogel [2]. On longer length scales, however, the behaviour crosses over to that of the $\operatorname{RFXY}$ model. We therefore predict that smectics pinned by such anisotropic weak disorder will exhibit the $X Y$ BG phase, with its universal disorder-induced logarithmic layer wandering character, $\overline{\left\langle(u(\boldsymbol{r})-u(0))^{2}\right\rangle}=C(d)(\ln r) / q_{0}^{2}$ [3]. However, unlike $3 D$ bulk smectics, which exhibit thermally driven $\ln r$ fluctuations, here $C(d)$ is universal, the logarithm persists for all of $2<d<4$, and the smectic layers are pinned. The immediate consequence is that $\mathrm{x}$-ray scattering will exhibit real-space power-law decay $\overline{\left\langle\rho_{G}(\boldsymbol{r}) \rho_{-G}(\mathbf{0})\right\rangle} \propto r^{-\eta(G)}$ with universal $\eta(G)$ exponents $\left(G=m q_{0}\right)$.

If, instead, the aerogel is uniaxially compressed, i.e., $\gamma>0$, we expect that one of the (previously soft) smectic $\boldsymbol{r}_{\perp}$-axes ( $x$ or $y$ ) will orient along the axis of compression $\hat{\boldsymbol{e}}_{h}$ (figure 1(b)). We denote this $\hat{e}_{h}$-directed axis as 'hard' $(h)$, and call the other $\perp$ axis, orthogonal to $\hat{e}_{h}$, the 'soft' $(s)$ axis, i.e., $\boldsymbol{r}_{\perp}=\left(\boldsymbol{r}_{h}, \boldsymbol{r}_{s}\right)$. The resulting effective Hamiltonian describing this system at long length scales is

$$
\begin{gathered}
H_{m=1}=\frac{1}{2} \int \mathrm{d}^{d} \boldsymbol{r}\left[\sum_{\alpha=1}^{n} K\left(\nabla_{\perp}^{2} u_{\alpha}\right)^{2}+B\left(\partial_{z} u_{\alpha}-\frac{1}{2}\left(\nabla_{\perp} u_{\alpha}\right)^{2}\right)^{2}\right. \\
\left.+\gamma\left(\nabla_{h} u_{\alpha}\right)^{2}-\sum_{\alpha, \beta=1}^{n} \frac{\Delta}{T}\left(\nabla_{\perp} u_{\alpha}\right) \cdot\left(\nabla_{\perp} u_{\beta}\right)\right]
\end{gathered}
$$

where we have neglected the positional random-field disorder, $\Delta_{V}$, which can be shown to be subdominant at long length scales [4]. $H_{m=1}$, equation (4), implies that the noninteracting propagator

$$
G_{\alpha \beta}(\boldsymbol{q}) \equiv V^{-1}\left\langle u_{\alpha}(\boldsymbol{q}) u_{\beta}(-\boldsymbol{q})\right\rangle_{0}=T G(\boldsymbol{q}) \delta_{\alpha \beta}+\Delta q_{\perp}^{2} G(\boldsymbol{q})^{2}
$$

with $G(\boldsymbol{q}) \equiv 1 /\left(K q_{\perp}^{4}+\gamma q_{h}^{2}+B q_{z}^{2}\right)$. As usual, at long length scales, the disorder $(\Delta)$ contribution to layer roughness dominates over the thermal $(T)$ part of $G_{\alpha \beta}(q)$. 
We first note that for vanishing strain $\gamma \rightarrow 0$, or equivalently at very short length scales, $H_{m=1}$ and the corresponding propagator reduce to those characterizing a smectic in unstrained isotropic aerogel [2]. The asymptotic long-length-scale behaviour of the full model described by $H_{m=1}$ is reached via two independent crossovers from the Gaussian, unstrained fixed point, during which the aerogel anisotropy $\gamma$ and the nonlinear elasticity become important. The qualitative form of this crossover is determined by the relative magnitudes of the corresponding bare couplings. For sufficiently weak strain $\left(\gamma<\gamma_{c}\right)$, the elastic anharmonicity becomes important first and this occurs at a crossover length scale

$$
\tilde{\xi}_{\perp}^{N L} \propto\left(\frac{K^{5 / 2}}{B^{1 / 2} \Delta}\right)^{1 /(5-d)}
$$

determined by the smectic in unstrained aerogel [2]. In this case the system first crosses over from the unstrained Gaussian to an unstrained anomalous fixed point. The final crossover to asymptotic strained anomalous behaviour takes place within the anomalously elastic smectic described by the wavevector-dependent elastic constants [2] and occurs at $\boldsymbol{q}_{\perp}$ such that $\tilde{K}\left(\boldsymbol{q}_{\perp}\right) q_{\perp}^{4} \approx \gamma q_{\perp}^{2}$, with $\tilde{K}\left(\boldsymbol{q}_{\perp}\right)$ calculated in reference [2], i.e. at $\xi_{\perp}^{c} \approx\left[K /\left(\gamma\left(\tilde{\xi}_{\perp}^{N L}\right)\right)^{\tilde{\eta}_{K}}\right]^{1 /\left(2-\tilde{\eta}_{K}\right)}$ (we use the tilde for exponents for isotropic disorder).

For the remainder of this paper we will focus on the other crossover scenario in which the strain $\gamma$ is sufficiently large $\left(\gamma>\gamma_{c}\right)$ that the crossover from Gaussian unstrained to Gaussian strained elasticity takes place at $\xi_{\perp}^{c}=\sqrt{ }(K / \gamma)$, before elastic nonlinearities become important. The critical value of $\gamma$ that demarcates between these two crossover scenarios is $\gamma_{c}=K /\left(\tilde{\xi}_{\perp}^{N L}\right)^{2}$.

For $\gamma>\gamma_{c}$, on length scales longer than $\xi_{\perp}^{c}=\sqrt{ }(K / \gamma)$ the effective Hamiltonian (and the propagator $G$ derived from it) is identical to that given in equation (4), but with all $\nabla_{\perp}$ replaced by $\nabla_{s}$, with $r_{s}$ a subset of $\boldsymbol{r}_{\perp}$-axes remaining soft even in the presence of aerogel anisotropy. Our goal then is to assess the role of elastic nonlinearities, at this new strained Gaussian fixed point, which become important beyond an even longer nonlinear crossover length scale $\xi_{s}^{N L}$ (along the 'soft' direction).

The length scale $\xi_{s}^{N L}$ can be determined from a simple perturbation theory in these nonlinear couplings of $H_{m=1}$, and is the length at which the effects of anharmonic elastic terms become significant. For $d<d_{u c}=7 / 2$, the corrections to the smectic elastic constants grow with system size and become significant for 'soft' length scales $>\xi_{s}^{N L}$, signalling the breakdown of conventional harmonic elasticity. We find

$$
\xi_{s}^{N L}=\left(\frac{2 \pi(7-2 d) K^{(7-d) / 2} \gamma^{(d-2) / 2}}{C_{d-1} \beta_{d-1} \Delta B^{1 / 2}}\right)^{1 /(7-2 d)} .
$$

The constant $C_{d}=2 \pi^{d / 2} /\left((2 \pi)^{d} \Gamma(d / 2)\right)$ and $\beta_{d}=\Gamma(d / 2) \Gamma(3-d / 2) / 2$. The corresponding lengths along the $z$ - and $h$-axes are given by $\xi_{z}^{N L}=\left(\xi_{s}^{N L}\right)^{2} / \lambda_{B}$ and $\xi_{h}^{N L}=$ $\left(\xi_{s}^{N L}\right)^{2} / \lambda_{\gamma}$, where $\lambda_{B} \equiv(K / B)^{1 / 2}$ and $\lambda_{\gamma} \equiv(K / \gamma)^{1 / 2}$.

To go beyond these crossover length scales $\xi_{z, h, s}^{N L}$ we use the renormalization group (RG) with an $\epsilon=7 / 2-d$ expansion. We find a nontrivial, glassy, $T=0$ fixed point, leading to strong disorder-generated power-law anomalous elasticity that dominates over the unmeasurably weak thermally generated (logarithmic) anomalous elasticity [6]. One consequence of the anomalous elasticity is that the long-length-scale elastic constants $K$, $B$ and the disorder variance $\Delta$ become wavevector dependent:

$$
\begin{aligned}
& K(\boldsymbol{k})=K k_{s}^{-\eta_{K}} f_{K}\left(k_{h} / k_{s}^{\zeta_{h}}, k_{z} / k_{s}^{\zeta_{z}}\right) \\
& B(\boldsymbol{k})=B k_{s}^{\eta_{B}} f_{B}\left(k_{h} / k_{s}^{\zeta_{h}}, k_{z} / k_{s}^{\zeta_{z}}\right) \\
& \Delta(\boldsymbol{k})=\Delta k_{s}^{-\eta_{\Delta}} f_{\Delta}\left(k_{h} / k_{s}^{\zeta_{h}}, k_{z} / k_{s}^{\zeta_{z}}\right)
\end{aligned}
$$


(note that $\gamma$ is not anomalous), with the anisotropy exponents $\zeta_{z} \equiv 2-\left(\eta_{B}+\eta_{K}\right) / 2$ and $\zeta_{h} \equiv 2-\eta_{K} / 2$. The exponents obey

$$
7-2 d+\eta_{\Delta}=\frac{\eta_{B}}{2}+\frac{7-d}{2} \eta_{K}
$$

exactly, due to the underlying exact rotational invariance of equation (4) about $\hat{e}_{h}$. To leading order in $\epsilon=7 / 2-d, \eta_{K}=16 \epsilon / 15=8 / 15, \eta_{B}=12 \epsilon / 15=2 / 5$, and $\eta_{\Delta}=2 \epsilon / 15=2 / 15$, the last equalities holding for $d=3(\epsilon=1 / 2)$. Since $\epsilon=1 / 2$ is quite small, we expect these exponents to be quantitatively accurate.

A different analytical continuation to $d$ dimensions, in which there are $d-2$ soft coordinates $\boldsymbol{r}_{s}$ and only a single hard axis, leads to an $\hat{\epsilon} \equiv 4-d$ expansion. The corresponding exponents are given by $\hat{\eta}_{K}=3 \hat{\epsilon} / 8=3 / 8, \hat{\eta}_{B}=3 \hat{\epsilon} / 4=3 / 4$, and $\hat{\eta}_{\Delta}=\hat{\epsilon} / 8=1 / 8$, with good agreement for $d=3$ (except for $\eta_{B}$ ) with the $\epsilon=7 / 2-d$ expansion results. The exact exponent relation for the $\hat{\epsilon}=4-d$ expansion is given by $4-d+\hat{\eta}_{\Delta}=\hat{\eta}_{B} / 2+2 \hat{\eta}_{K}$ and reassuringly agrees with equation (9) for $d=3$.

Further accuracy can be gained by weighted averaging of the $7 / 2-d$ and $4-d$ expansions, according to $\eta_{K, \Delta} \rightarrow\left(4 \eta_{K, \Delta}+\hat{\eta}_{K, \Delta}^{s}\right) / 5$. The factor of 4 reflects the higher accuracy of the $7 / 2-d=\epsilon$ expansion. The prediction for $\eta_{B}$ is then made using the exact scaling relation, giving, for $d=3, \eta_{K}=0.50, \eta_{B}=0.26$, and $\eta_{\Delta}=0.13$.

We now study the translational and orientational order in the presence of this strong disorder-driven anomalous elasticity. The former is characterized by the growth of smectic layer roughness with, e.g., $r_{s}$ :

$C\left(r_{s}\right) \equiv \overline{\left\langle\left(u\left(r_{s}, 0_{h}, 0_{z}\right)-u\left(0_{s}, 0_{h}, 0_{z}\right)\right)^{2}\right\rangle}=\int \frac{\mathrm{d}^{3} k}{(2 \pi)^{3}} 2\left(1-\cos \left(k_{s} r_{s}\right)\right) \Delta(\boldsymbol{k}) k_{\perp}^{2} G^{2}(\boldsymbol{k})$

from which the translational correlation length $\xi_{s}^{X}$ (the inverse of the x-ray diffraction peak width), can be computed via the condition $C\left(r_{s}=\xi_{s}^{X}\right) \equiv a^{2}$, with $a$ the smectic layer spacing. $\xi_{s}^{X}$ is determined by the relative order of many crossover length scales. For $\xi_{s}^{X}<\xi_{\perp}^{c}$, $\xi_{s}^{X}$ is identical to that due to isotropic disorder [2]. For $\xi_{s}^{X}>\xi_{\perp}^{c}$, there are three possibilities, depending on whether the anomalous elasticity sets in before or after the layer roughness reaches $a$, and whether the isotropic-to-anisotropic crossover takes place near the harmonic or the anomalous elastic fixed point:

$$
\xi_{s}^{X}= \begin{cases}\xi_{s}^{N L}\left(\frac{a}{\lambda_{B}}\right)^{2} & \xi_{\perp}^{c}<\xi_{s}^{X}<\xi_{s}^{N L}, \gamma>\gamma_{c} \\ \xi_{s}^{N L}\left(\frac{a}{\lambda_{B}}\right)^{2 /\left(\eta_{B}+\eta_{K}\right)} & \xi_{\perp}^{c}<\xi_{s}^{N L}<\xi_{s}^{X}, \gamma>\gamma_{c} \\ \xi_{\perp}^{c}\left(\frac{a}{\lambda_{B}}\right)^{2 /\left(\eta_{B}+\eta_{K}\right)}\left(\xi_{\perp}^{c} / \tilde{\xi}_{\perp}^{N L}\right)^{\left(\tilde{\eta}_{B}+\tilde{\eta}_{K}\right) /\left(\eta_{B}+\eta_{K}\right)} & \tilde{\xi}_{\perp}^{N L}<\xi_{\perp}^{c}<\xi_{s}^{X}, \gamma<\gamma_{c} .\end{cases}
$$

From these correlation lengths we see that it is the ratio $a / \lambda_{B}$ which determines whether $\xi_{s}^{X}$ lies in a length-scale regime in which anharmonic effects are important. For small $B, \lambda_{B} \gg a$, and anharmonic effects are unimportant. Note also that in the strained-length-scale regime, $\xi_{s}^{X}$ will depend on $B, K, \Delta$, and $\gamma$. Thus, one could test the predictions of equation (11) by measuring the dependence of $\xi_{s}^{X}$ on the strength of the compression (i.e., $\gamma$ ) which could be adjusted directly. In all length-scale regimes, the x-ray correlation length is finite even as $T \rightarrow 0$, signalling the destruction of the conventional (quasi-) long-ranged translational smectic order.

As emphasized in reference [2], this lack of translational order does not imply that the low-temperature phase replacing the smectic is simply nematic (or isotropic). Our 
detailed calculations [4] indicate that despite the lack of (quasi-) long-ranged smectic order, dislocation loops remain bound for weak anisotropic disorder, and therefore the low temperature phase replacing the smectic must be distinct from the nematic, separated from it by a thermodynamically sharp dislocation-unbinding phase transition. We call this low-temperature phase the ' $m=1$ Bragg glass'.

The stability of this exotic glass phase is contingent upon our implicit assumption of long-ranged orientational (nematic) order. That this assumption is valid can be easily seen by computing $\overline{\left\langle|\delta n|^{2}\right\rangle}=\overline{\left\langle|\nabla u|^{2}\right\rangle}$, and taking into account the wavevector-dependent elastic moduli $K(\boldsymbol{k})$ and $B(\boldsymbol{k})$ and disorder variance $\Delta(\boldsymbol{k})$, as given by equations (6), (7), and (8). There are unstrained and strained contributions to $\overline{\left\langle|\delta \boldsymbol{n}|^{2}\right\rangle}$, arising from modes with $q_{\perp}>1 / \xi_{\perp}^{c}$ and $q_{\perp}<1 / \xi_{\perp}^{c}$, respectively. Using the corresponding anomalous exponents $\eta_{K}, \eta_{B}, \eta_{\Delta}$ [2] in the computation of the strained and unstrained parts, for finite strain $\gamma$ and weak disorder $\Delta$, we indeed find long-ranged orientational order. In the weak-strain limit $\left(\gamma<\gamma_{c}\right)$, the unstrained part dominates, in 3D scaling in a universal way with strain $\gamma$ and disorder $\Delta$ as $\left(\Delta^{\mu} / \gamma^{\mu-1}\right)$, where $\mu=\tilde{\eta}_{B} /\left(2-\tilde{\eta}_{K}\right)$. Using reference [2], we estimate $\mu$ to be $3 / 2$. Since $\overline{\left\langle|\delta \boldsymbol{n}|^{2}\right\rangle}$ can therefore get arbitrarily large at small $\gamma$ and large $\Delta$, we expect our system to be in the orientationally disordered liquid phase in this range of parameters. On the other hand, for large $\gamma$ and small $\Delta$ the system will exhibit long-ranged orientational order and will therefore be in the $m=1 \mathrm{BG}$ phase (as in figure 2(a)). In analogy with the Lindemann criterion for melting, the phase boundary is roughly determined by the condition $\overline{\left\langle|\delta \boldsymbol{n}|^{2}\right\rangle} \approx \mathrm{O}(1)$. This leads to the phase boundary quoted in equation (1), and shown in figure 2(a).

On the other hand, rather than rely on the untrustworthy (in 3D) $5-\epsilon$ expansion, which predicts no SBG for isotropic $(\sigma=0)$ disorder [2], we can infer the topology of the phase diagram on the basis of preliminary experimental evidence [5], which suggests the stability of the SBG for weak isotropic disorder. This suggests that the $m=1 \mathrm{BG}$ extends all the way down to vanishing strain, $\sigma>0$, as illustrated in figure 2(b).

Light scattering, which measures the director correlation $\overline{\left\langle\delta n_{i}(\boldsymbol{q}) \delta n_{j}(-\boldsymbol{q})\right\rangle}$ provides an independent means of testing the predictions of the theory. Finally, since anomalous elasticity also implies a nonlinear stress-strain relation at arbitrarily weak stress, our predictions for it can be independently probed in an a.c. acoustic experiment, searching for an unusually large second-harmonic response.

\section{Acknowledgments}

LR and BJ acknowledge support by the NSF DMR-9625111, the MRSEC DMR-9809555, and the A P Sloan and David and Lucile Packard Foundations. JT and KS were supported by the NSF DMR-9634596.

\section{References}

[1] Crawford G P and Žumer S (ed) 1996 Liquid Crystals in Complex Geometries (London: Taylor and Francis)

[2] Radzihovsky L and Toner J 1999 Phys. Rev. B 60206 Radzihovsky L and Toner J 1997 Phys. Rev. Lett. 784414 Radzihovsky L and Toner J 1997 Phys. Rev. Lett. 794214

[3] Giamarchi T and Le Doussal P 1994 Phys. Rev. Lett. 721530 Fisher D S 1997 Phys. Rev. Lett. 781964

[4] Saunders K, Radzihovsky L and Toner J, unpublished

[5] Bellini T, Clark N, Radzihovsky L and Toner J, unpublished

[6] Grinstein G and Pelcovits R A 1981 Phys. Rev. Lett. 47856 Golubovic L and Wang Z G 1992 Phys. Rev. Lett. 692535 